\title{
Klimawandel und Bachforellenrückgang - gibt es einen Zusammenhang? Resultate aus der Schweiz
}

\author{
Patricia Burkhardt-Holm
}

Eingegangen: 31. Oktober 2008/Akzeptiert: 23. Februar 2009/Online veröffentlicht: 20. März 2009

(C) Springer-Verlag 2009

Zusammenfassung Hintergrund und Ziel Eine Abnahme der Forellenfangerträge in den Schweizer Fliessgewässern um 60\% in den Jahren 1980-2000 war Anlass für eine schweizweite Suche nach den möglichen Ursachen. Im Rahmen des dafür eingerichteten Projekts „Fischnetz“ wurden 12 Hypothesen untersucht. Eine davon nimmt an, dass sich die globale Klimaveränderung nachteilig auf die Bachforellen der Schweizer Fliessgewässer auswirkt. Hier wird dargestellt, welche Parameter sich verändert haben und welche Konsequenzen der einheimischen Bachforelle daraus entstehen könnten.

Material und Methoden Die vorhandene Literatur wird analysiert und eine Synthese versucht, die die Zusammenhänge zwischen den Faktoren in ihrer Wirkung untereinander und auf den Fisch veranschaulicht.

Ergebnisse In den vergangenen 25 Jahren ist die Durchschnittstemperatur in den Fliessgewässern der Schweiz um etwa $1{ }^{\circ} \mathrm{C}$ gestiegen. Es gibt Hinweise, dass dieser Temperaturanstieg eine Vorverschiebung des Schlüpfzeitpunktes und eine Habitatverlagerung um 130 Höhenmeter flussaufwärts zur Folge hat. Häufigere Perioden mit Wassertemperaturen über $15^{\circ} \mathrm{C}$ während 2-4 Wochen werden vermehrte klinische Ausbrüche der Nierenkrankheit PKD mit sich bringen. Das Niederschlagsmuster änderte sich und resultiert bereits in vermehrten Winterhochwassern und Erosion. Letztere trägt zu einem erhöhten Eintrag von Feinsedimenten bei, die

Herrn Prof. Dr. Dr. h. c. Volker Storch zum 65. Geburtstag gewidmet. Herausgeber: Henner Hollert · Thomas Braunbeck

P. Burkhardt-Holm $(\bowtie)$

Mensch-Gesellschaft-Umwelt MGU,

Universität Basel,

Vesalgasse 1, 4051 Basel, Schweiz

E-Mail: patricia.holm@unibas.ch einerseits die Gesundheit juveniler Bachforellen beeinträchtigen und andererseits durch verstärkte Sohlenkolmation die Reproduktionsleistung vermindern können.

Diskussion Die Konsequenzen der beobachteten Veränderungen sind im Kontext mit anderen anthropogenen Einflüssen zu betrachten. Diese stellen zusätzliche Erschwernisse in der Einschätzung der Bedeutung der Klimaerwärmung auf Bachforellen dar. Wasserentnahmen, bzw. Rückgabe des erwärmten Wassers, tragen zur Temperaturerhöhung der Flüsse bei. Stauhaltungen wirken sich ebenfalls auf Temperaturprofile und Schwebstofffrachten der Flüsse aus, Begradigungen und Fragmentierungen fördern die Kolmation und verhindern das Ausweichen der Fische in kühlere Lebensräume.

Schlussfolgerungen Mit ansteigenden Wassertemperaturen ist eine weitere Verkleinerung der Lebensräume von Fischarten sommerkühler Gewässer sowie die Zunahme temperatursensitiver Fischkrankheiten zu erwarten.

Empfehlungen und Perspektiven Massnahmen, die den ungünstigen Konsequenzen der Klimaveränderungen entgegen wirken können, werden empfohlen. Hierzu gehören die Verbesserung und Vernetzung der Lebensräume der Fische sowie eine Verbreiterung und Bestockung des Uferstreifens.

Schlüsselwörter Abfluss · Bachforelle $\cdot$ Entwicklung · Feinsediment $\cdot$ Fischgesundheit $\cdot$ Habitat $\cdot$ Klimaveränderung $\cdot$ Kolmation $\cdot$ Reproduktion $\cdot$ Wassertemperatur

\section{Climate change and decline in abundance of brown trout - is there a link? Results from Switzerland}

Abstract Background, aim, and scope In response to a $60 \%$ decrease in brown trout catch between 1980 and 2000, a Swiss-wide search was initiated to investigate possible 
causes. The project, named 'Fischnetz' (fishing net), investigated 12 hypotheses. One of those suggested a detrimental effect of global climate change. I report here which parameters changed and what the possible consequences for native brown trout are.

Materials and methods The literature is critically analysed and results are synthesised to show the interactions between different climatic factors and their effects on fish.

Results In the last 25 years, an increase in temperature by approximately $1{ }^{\circ} \mathrm{C}$ was indicated in the rivers of Switzerland. This is associated with an earlier emergence of trout from the gravel. Warming results in an upward shift of the preferred thermal habitats. Furthermore, an increase in the clinical outbreak of the Proliferative Kidney Disease PKD (for which a temperature of $15^{\circ} \mathrm{C}$ for more than 2-4 weeks is necessary) can be recorded. The precipitation pattern changed and an intensification of high floods in winter results in higher erosion. This can in turn lead to an increased level of fine sediments which may affect health of juvenile brown trout and is assumed to reduce reproduction success. Discussion The consequences of climate change are discussed in concert with other anthropogenic factors. Storage reservoirs, as well as water withdrawal (and return of heated water, respectively) affect temperature profiles and sediment load. Fragmentation, channelization and straightening of rivers accelerate clogging and restrain fish from upward migration and evacuation to more suitable habitats.

Conclusions With increasing temperatures a downsizing of habitats of cold-water fish species, as well as an increase of diseases which are temperature sensitive, is assumed.

Recommendations and perspectives Mitigation measures which lead to a morphological improvement of river systems, such as river widening and improvement of the connectivity between river stretches and their tributaries, as well as improving river bank vegetation, are recommended.

Keywords Brown trout $\cdot$ Clogging - Development · Discharge $\cdot$ Fine sediments $\cdot$ Fish health $\cdot$ Habitat $\cdot$ Climate change $\cdot$ Reproduction $\cdot$ Water temperature

\section{Problemstellung}

Die Bachforelle (Salmo trutta) ist die am weitesten verbreitete Fischart in der Schweiz (Zaugg et al. 2003). Sie ist auch bei den Anglern sehr beliebt und ist die am häufigsten gefangene Art in der Freizeitfischerei. Darauf basierend verfügt die Schweiz - wo jeder gefangene Fisch seit Jahren registriert werden muss - über sehr ausführliche Daten zum Bachforellenfang. Auf dieser Grundlage konnte deshalb seit den 1970er-Jahren ein starker Rückgang bei den Bachforellenfängen verzeichnet werden. Zwar gibt es eine Abnahme auch bei anderen Arten, doch diese sind stark durch die Rah- menbedingungen der jeweiligen Gewässer geprägt, während die Bachforelle flächendeckend verbreitet und so besonders ins Zentrum des Interesses für überregionale Fragen nach den Ursachen für Fangrückgänge gerückt ist.

Zwischen 1980 und 2001 nahm die Zahl geangelter Bachforellen von ca. 1,5 Mio. auf weniger als 0,5 Mio. ab. Dabei ist der Rückgang der Fänge regional unterschiedlich stark ausgeprägt, und er beginnt in den verschiedenen Gewässern zu unterschiedlichen Zeitpunkten. Untersuchungen zur Fischgesundheit wiesen zudem auf gesundheitliche Beeinträchtigungen hin. Dies alles veranlasste verschiedene Behörden, WissenschaftlerInnen, Fischereivereine und die chemische Industrie dazu, ein schweizweites Projekt zu lancieren, in dem die Sachverhalte dokumentiert, die Ursachen ergründet und Massnahmenvorschläge entwickelt wurden. Dieses 5-jährige Projekt namens „Fischnetz“ umfasste 77 Teilprojekte, in denen mehr als 100 WissenschaftlerInnen und hochschulexterne ExpertInnen aktiv zum Erfolg des Projektes beigetragen haben. Ein guter Indikator für die breite Akzeptanz des Projektes - aber auch die damit verbundene Erwartungshaltung - war die finanzielle Unterstützung: Alle 26 Kantone der Schweiz und das Fürstentum Liechtenstein halfen, neben den Bundesinstitutionen Eawag und Bundesamt für Umweltschutz (Bafu), dem Schweizerischen Fischereiverband und der Schweizerischen Gesellschaft für chemische Industrie (SGCI) substantiell bei der Finanzierung (Fischnetz 2004).

Naturgemäss war der umfangreichste Teil des Projektes die Ursachensuche. Hierfür wurden 12 Hypothesen aufgestellt und in verschiedene Forschungsfragen aufgegliedert, die wiederum in den erwähnten Teilprojekten bearbeitet wurden (Burkhardt-Holm et al. 2002). Als wesentlichste Resultate sind festzuhalten: Ein Teil des Fangrückgangs ist auf die abgenommene Fangintensität zurückzuführen, ein anderer Teil auf die verringerten Fischbestände. Dieser Bestandesrückgang hat verschiedene Ursachen, schweizweit am wichtigsten schlägt eine Krankheit, die Proliferative Nierenkrankheit PKD (s. unten) und schlechte Lebensraumqualität, sowohl bezüglich Wasser- als auch Habitatsqualität, zu Buche (Burkhardt-Holm et al. 2005). Die Auflösung der Komplexität der dynamischen Zusammenhänge zwischen den verschiedenen, sich gegenseitig - und die Forellen direkt und indirekt beeinflussenden Faktoren stellte eine der grössten wissenschaftlichen Herausforderungen dar. Im vorliegenden Beitrag soll dies am Beispiel der Klimaveränderung näher ausgeführt werden.

Eine Hypothese war, dass sich die globale Klimaveränderung nachteilig auf die Bachforellen auswirkt. Wir wissen, dass die Lufttemperatur in der Schweiz stärker zugenommen hat als in anderen nord- und westeuropäischen Ländern $\left(0,57^{\circ} \mathrm{C}\right.$ pro Dekade gegenüber $0,24^{\circ} \mathrm{C}$ pro Dekade im Schnitt in der nördlichen Hemisphäre; Hari et al. 2006). Diese Zunahme spiegelt sich in der Wassertemperatur sehr 
gut wieder (Mohseni und Stefan 1999). Darüber hinaus ist bekannt, dass sich mit ansteigender Temperatur kälteliebende Fischarten weiter nach Norden zurückziehen (Perry et al. 2005).

$\mathrm{Zu}$ unserer Annahme lässt sich im Detail fragen (1) ob und wie stark sich die Wassertemperatur und andere Parameter des Klimas, die sich auf Fische auswirken können, geändert haben und (2) ob diese Veränderungen ausreichend sind, um nachweisbare Effekte in Bachforellenpopulationen hervorzurufen.

\section{Die Biologie der Bachforelle - klimarelevante Parameter}

Voraussetzung zur Beantwortung der angesprochenen Fragen ist eine ausreichende Kenntnis über die Biologie der Bachforelle und ihre Ansprüche an Temperatur und Lebensraum. Salmo trutta ist die Leitfischart der Forellenregion, die sich auszeichnet durch hohe Fliessgeschwindigkeit sauerstoffreicher, kiesiger, sommerkühler Gewässer. Als adultes Tier zeigt die einheimische Bachforelle optimale Wachstumsraten in einem Temperaturbereich zwischen 4 und $19^{\circ} \mathrm{C}$ (Elliott 1981). Ober- und unterhalb dieser Temperaturen werden Wachstum und Nahrungsaufnahme reduziert und mit zunehmender Entfernung vom Optimum schliesslich eingestellt (Reichenbach-Klinke 1976). Die Brütlinge sind noch empfindlicher gegenüber warmen Temperaturen; ihr Wachstumsoptimun liegt zwischen 6 und $14^{\circ} \mathrm{C}$ (Schmeing-Engberding 1953). Diesen meist in Laborversuchen ermittelten Werten stehen die tatsächlich im Freiland gefundenen Präferenzen gegenüber, die ermittelt wurden, indem die Fangzahlen mit den Wassertemperatu-

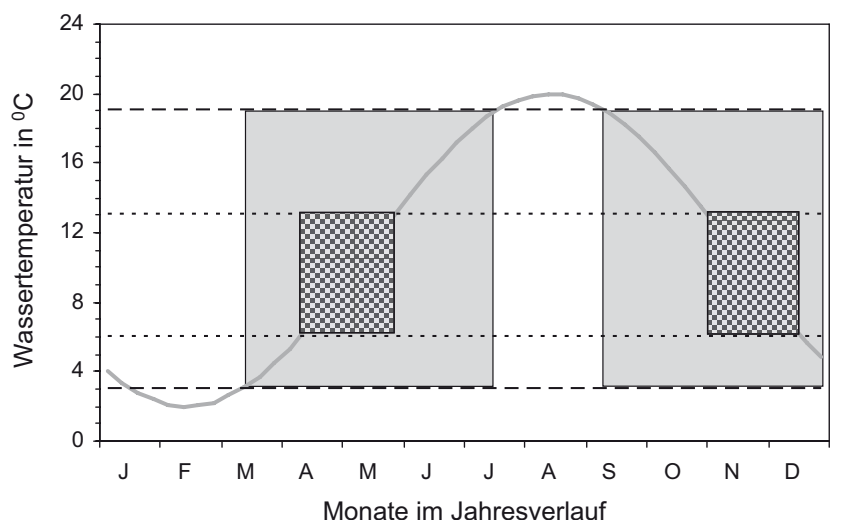

Abb. 1 Veränderung der Wassertemperatur in einem typischen Fliessgewässer der Schweiz im Jahresverlauf in der Periode 1987-2002 (Kurve). Die Periode des optimalen Wachstums im ersten (gewürfelt) und in allen weiteren (grau) Lebensjahren der Bachforelle als Blöcke dargestellt, nach Schmeing-Engberding (1953) und Hari et al. (2006) (verändert) ren verglichen wurden. Demnach ist in den schweizerischen Gewässern die obere Wachstumsgrenze für die Nachkommen bei $13^{\circ} \mathrm{C}$ erreicht, für die adulten bei $19,1^{\circ} \mathrm{C}$ (Hari et al. 2006; Abb. 1). Im Winter stellen die Tiere ihr Wachstum unterhalb von 2,5 bzw. $3,9^{\circ} \mathrm{C}$ ein (Hari et al. 2006). Kurzzeitigen Abweichungen kann durch Ausweichen in kühlere Habitate, wie Pools oder Stellen mit (Grund)wassereinstrom, begegnet werden, falls dies die Habitatvielfalt erlaubt (Peter 1998).

Der Kies des Bachbetts muss ebenfalls den Ansprüchen der Bachforelle genügen, damit die Tiere sich erfolgreich fortpflanzen können: Die laichreifen Weibchen schlagen an gut durchströmten Stellen im freibeweglichen Kies Laichgruben. Die dort zwischen Oktober bis Januar abgelegten Eier werden dann wieder mit Kies überdeckt, womit sie vor Verdriftung durch Hochwasser oder Beschädigung durch Geschiebe sicher sind. Wenn das Bachbett stark mit Sedimenten verstopft (,kolmatiert") ist, werden die Eier entweder nicht abgegeben oder nur unzureichend mit Kies geschützt (Crisp 2000). Die Entwicklung des Embryos ist temperaturabhängig; es wird mit 420 Tagesgraden gerechnet, also 420 Tage bei $1{ }^{\circ} \mathrm{C}$, bzw. entsprechend weniger bei höheren Temperaturen. Die Embryos schlüpfen als Dottersacklarven und bleiben im Interstitial, bis der Dottersack aufgebraucht ist. Während dieser Inkubationszeit brauchen die Eier, und später die Brütlinge, sauberes, rasch fliessendes Wasser, das die optimale Sauerstoffversorgung und den Abtransport von ausgeschiedenen Stoffen gewährleistet. Ereignisse wie eine frühzeitige Schneeschmelze und gehäufte stärkere oder lang anhaltende Regenfälle, die vor allem in der vegetationsärmeren Jahreszeit mit einer erheblichen Erosion einhergehen können, wirken sich dabei besonders fatal aus. Eine Anreicherung von Feinsediment in der Bachsohle kolmatiert das Kiesbett und behindert somit den Abtransport von ausgeschiedenen Stoffen und die Zufuhr von Sauerstoff bei den Eiern bzw. den Brütlingen (Greig et al. 2007).

Nach der Emergenz aus dem Kies suchen die jungen Bachforellen unterschiedliche Areale in einem Fluss auf, ihre Präferenzen wechseln je nach Tageszeit, Jahreszeit und Aktivität (Roussel und Bardonnet 2002). Sie sind anfällig für Trübung, da feine Sedimentpartikel die Kiemen verstopfen oder die Epithelien schädigen können (Berry et al. 2003).

Kritisch für das Überleben der jungen Sömmerlinge sind die Temperaturverhältnisse besonders im ersten Sommer. Die proliferative Nierenkrankheit (Proliferative Kidney Disease, PKD), zum Beispiel, kann hohe Sterblichkeiten bis zu 100\% verursachen (Feist und Bucke 1993). Der klinische Ausbruch der Krankheit ist temperaturabhängig und erfolgt erst, wenn eine Wassertemperatur von $15^{\circ} \mathrm{C}$ mehr als 2-4 Wochen überschritten wird (Schubiger et al., in Vorbereitung). 


\section{Auswirkung der Klimaveränderung auf Temperatur, Abfluss und Feinsedimenteintrag in Fliessgewässern}

\subsection{Veränderung der Wassertemperaturen in Schweizer Fliessgewässern}

Seit den 1960ern werden in der Schweiz regelmässig Temperatur- und Abflussdaten an Flüssen und Seen erhoben. Gute, nahezu ununterbrochene Datenreihen liegen für 25 Stellen für den Zeitraum von 1978-2002 vor, sie umfassen eine Höhendifferenz von $4607 \mathrm{~m}$. Parallel dazu wurden Fangdaten der Bachforelle ausgewertet, und zwar von 413 Flussabschnitten, die eine Höhendifferenz von 193-3029 m umfassen. Die Temperaturverläufe der meisten untersuchten Fliessgewässer, sowohl im Mittelland als auch in den Voralpen und Alpen, zeigen einen Trend zu höheren Temperaturen von bis $\mathrm{zu}+1,1^{\circ} \mathrm{C}$, während dieser Anstieg weniger deutlich in Flüssen ausfällt, die durch Gletscher oder Wasserkraftwerke beeinflusst werden (Hari et al. 2006, Matulla et al. 2007). Ein sprunghafter Anstieg wurde 1987/88 verzeichnet, der vermutlich mit der gleichzeitig beobachteten Veränderung beim NAO-Index (North Atlantic Oscillation) zusammenhängt. Der NAO charakterisiert die bei uns auf das Klima einwirkenden Luftdruckverhältnisse im Nordatlantik. Der Temperaturanstieg ist nicht gleichmässig über das ganze Jahr festzustellen, sondern betrifft vor allem die Monate Januar bis August, während im Herbst keine Veränderung zu konstatieren ist. Die höheren Temperaturen werden im Jahresverlauf um 5-7 Tage früher erreicht, im Frühjahr beträgt diese Vorverschiebung sogar 14 Tage (Hari et al. 2006).

\subsection{Veränderung der Häufigkeit \\ und Stärke von Winterhochwassern}

Da die Erbrütung der Bachforellen im Kies während des Winters erfolgt, ist auch einer möglichen Zunahme von Winterhochwassern Aufmerksamkeit zu zollen, insbesondere wenn diese mit erhöhtem Geschiebetrieb, einem Aufreissen der Flusssohle und damit einer Zerstörung der Laichgruben einhergehen. Eine vergleichende Trendanalyse zum Abflussregime in 48 Einzugsgebieten in der Schweiz zeigt, dass der jährliche Abfluss im letzten Jahrhundert zugenommen hat, vor allem bedingt durch höheren Abfluss in Winter, Herbst und Frühling (Birsan et al. 2005). Klimaprognosen sagen eine Verschiebung der Hochwasser vom Sommer in den Winter voraus, und besonders im Alpenvorland der Nordschweiz sind winterliche Hochwasser häufiger zu erwarten (Bader und Kunz 1998, Schädler 2000). Mit dem erwarteten Temperaturanstieg wird vorhergesagt, dass die Frühlingshochwasser auf der Alpennordseite um 0,5-2 Monate pro Jahrhundert und auf der Alpensüdseite um zwei
Monate früher auftreten, jedoch in einer um $20-50 \%$ verminderten Stärke (Jasper et al. 2004; Horton et al. 2006). Es wurde daraufhin von uns im Fischnetz untersucht, ob wir in der Schweiz eine Zunahme der Winterhochwasser feststellen können. In ihrer Studie stellte Santschi (2003) fest, dass in $35 \%$ der untersuchten 41 Flüsse im Schweizer Mittelland und Jura in den letzten 40 Jahren eine Zunahme der Winterhochwasser zu verzeichnen war. In einzelnen Einzugsgebieten wie der Emme hat sowohl die Höhe als auch die Häufigkeit der mittleren und niedrigen Winterhochwasser zugenommen (Santschi 2003).

\subsection{Veränderung der Erosion und Einträge von Feinsedimenten in die Fliessgewässer}

Klimabedingte Veränderungen, die eine Erosionszunahme und in dessen Folge eine Erhöhung des Eintrags von Feinsedimenten in die Fliessgewässer hervorrufen, sind auf Form, Stärke und Häufigkeit der Niederschläge hin zu untersuchen. Im Hinblick auf das Niederschlagsmuster in den Alpen wird ein negativer Trend südlich der Alpen verzeichnet, während nördlich der Alpen, besonders in Winter und Frühling, eine Zunahme in Häufigkeit und Stärke der Niederschläge, v. a. der Starkregenereignisse, festgestellt wird (Brunetti et al. 2006). Letzteres trägt massgeblich zu erhöhter Erosion bei. Darüber hinaus haben die Schneehöhen in niedrigen Höhenlagen in den letzten 20 Jahren abgenommen und die Schneeschmelze tritt im Frühjahr um ein bis zwei Wochen früher auf. Es wird angenommen, dass sich dieser Trend verstärken und die Dauer der Schneebedeckung um mehr als 100 Tage abnehmen wird (Jasper et al. 2004; Horton et al. 2006). Höhere Niederschläge in Form von Regen und veränderte Schneebedeckung lassen, besonders zu Zeiten, in denen der Boden nicht durch Vegetation geschützt wird, eine weitere Zunahme der Bodenerosion erwarten. Erosion verstärkt den Eintrag von feinen Bodenpartikeln in die Gewässer, wobei Fliessgewässer, aufgrund ihrer hohen relativen Uferlänge, besonders betroffen sind. Es wird angenommen, dass die Sedimentlast im alpinen Rheineinzugsgebiet bis zum Jahr 2100 um mehr als 200\% zunehmen wird (Asselman et al. 2003).

Im freien Wasserkörper halten sich Schwebstoffe je nach Beschaffenheit, Grösse und Hydraulik unterschiedlich lange auf, bevor sie in langsamer fliessenden Streckenabschnitten sedimentieren. Höhere Sedimenteinträge sind meist an Hochwasser gekoppelt und treten während relativ kurzer Zeitspannen auf, oft während Schneeschmelze und starken Niederschlägen (Asselman et al. 2003, Fischnetz 2004). Für Spitzenhochwasser wurde in der Donau eine Verdopplung der Konzentration suspendierter Sedimente beschrieben (Summer et al. 1994), während andere Studien keine klaren Trends feststellen konnten (Zobrist et al. 2004). Die gemessenen Konzentrationen an suspendierten Schwebstoffen in 
Schweizer Fliessgewässern erreichen bei Punktmessungen selten mehr als $10.000 \mathrm{mgl}^{-1}$; in 14-Tage-Proben $(80 \%$ Perzentil) werden zwischen 300 und $1.000 \mathrm{mgl}^{-1}$ gemessen (Zobrist et al. 2004; unveröffentlichte Daten des Bundesamtes für Wasser und Geologie (BWG)) wobei die hohen Werte hauptsächlich in Flüssen mit alpinem Einzugsgebiet auftreten (Fischnetz 2004). Bezüglich Partikelform und -grösse, sowie ihrer geochemischen Beschaffenheit, ist für Sedimente in Fliessgewässern des alpinen Raums wenig bekannt.

Eine erhöhte Ablagerung der Sedimente behindert die Sauerstoffversorgung im Interstitial und kann zur Kolmation führen. Eine Zunahme der Kolmation wurde in verschiedenen Ländern festgestellt (Berry et al. 2003; Fischnetz 2004), sie ist jedoch methodisch sehr schwer zu fassen - zumal sie sich rasch durch Hochwasser verändern kann (Scheurer et al. 2009).

\section{Ergebnisse: Konsequenzen der Klimaveränderung für die Bachforelle in Schweizer Fliessgewässern (Abb. 2)}

\subsection{Temperaturerhöhung}

Für alle biochemischen Prozesse gilt die Temperatur-Geschwindigkeitsregel, die besagt, dass sich mit einer Temperaturerhöhung um $10^{\circ} \mathrm{C}$ alle entsprechenden Prozesse verdoppeln. Bei Fischen kann sich die Entwicklungsrate sogar um bis das Fünffache steigern, wenn die Temperatur um $10^{\circ} \mathrm{C}$ ansteigt (Rombough 1997). Den positiven Effekten - Stoffwechselprozesse laufen schneller ab, Wachstum und Entwicklung erfolgen rascher - stehen allerdings ne- gative Effekte - Erhöhung der Atemfrequenz, damit vermehrte Aufnahme von Schadstoffen - gegenüber. Indirekte Wirkungen auf die Verfügbarkeit der Nahrungsorganismen, die Entwicklung und Infektiosität der Krankheitserreger, und chemische Prozesse im Wasser, sowie interspezifische Konkurrenz sind zu beachten und bisher noch kaum untersucht. Eine verallgemeinernde Aussage bezüglich des Überwiegens positiver oder negativer Effekte ist daher nicht möglich.

Durch die höheren Temperaturen und die Vorverschiebung des Frühlings wird der Schlüpfzeitpunkt früher erreicht. Gemäss unserer Daten ergibt sich eine Verkürzung der Entwicklung von früher 91 Tagen auf heute 77 Tage (Hari et al. 2006). Die Konsequenzen für die Bachforelle sind schwer abschätzbar. Einerseits entwickelt sich auch die Natur (z. B. Futtertiere) schneller, andererseits wirken noch andere Faktoren, wie Tageslänge, die nicht mit der Temperatur korrelieren.

\subsection{Temperaturabhängige Krankheiten}

Einer der indirekten Effekte, die die Temperaturerhöhung mit sich bringt, ist die zunehmende Ausbreitung temperaturabhängiger Krankheiten. Die Proliferative Nierenkrankheit PKD wird durch den Parasiten Tetracapsuloides bryosalmonae hervorgerufen. Dieses Myxozoon infiziert die Bachforellen bei Temperaturen oberhalb von $8^{\circ} \mathrm{C}$; ein klinischer Krankheitsausbruch erfolgt aber erst, wenn die Wassertemperatur 2-4 Wochen lang einen Tagesmittelwert von $15^{\circ} \mathrm{C}$ übersteigt. Besonders betroffen sind Sömmerlinge. In zwei Flüssen der Schweiz konnte die Abhängigkeit der

\begin{tabular}{|llll}
\hline Adulte im Laichgeschäft & Eier in Laichgrube & Dottersackbrütlinge & Brütlinge \\
\hline
\end{tabular}

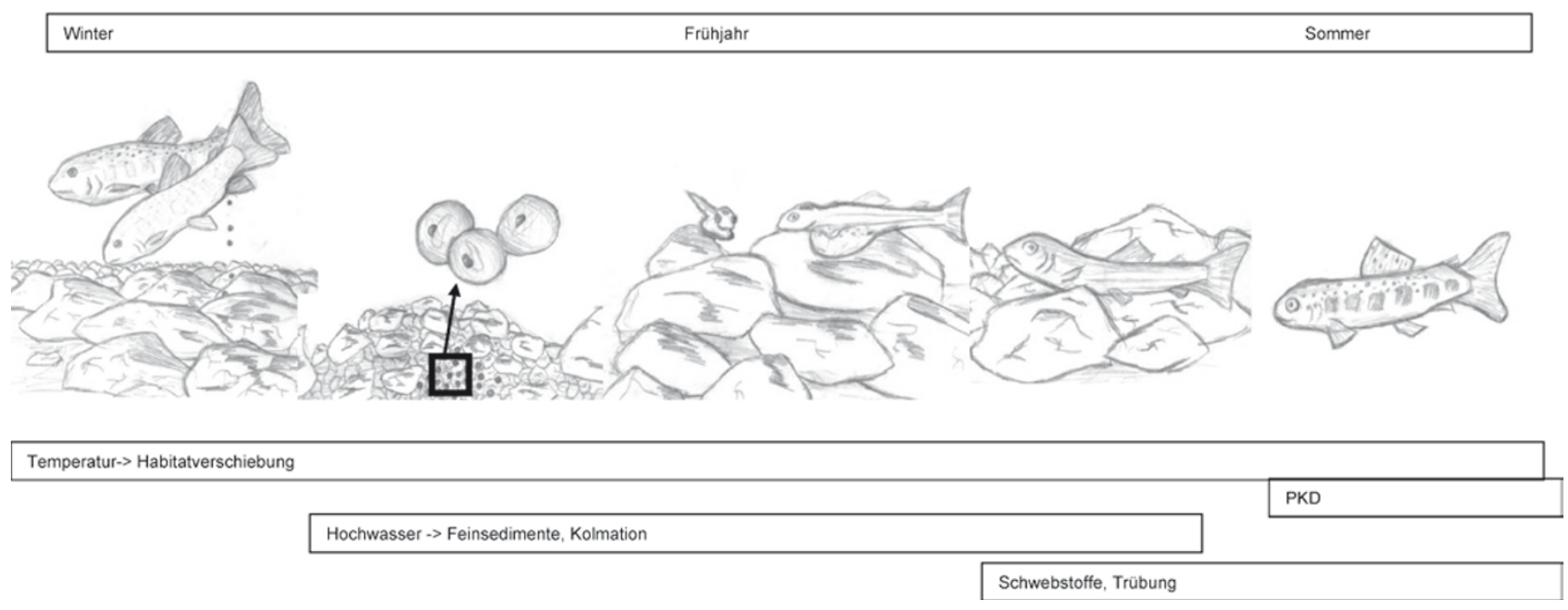

Abb. 2 Wirkung der Klimaveränderung auf die Lebensstadien der Bachforelle (prioritär beeinflussende Faktoren; Zeichnung: Fynn Holm) 
Sömmerlingsmortalität von der Wassertemperatur erstmals überzeugend im Freiland gezeigt werden: In der kühleren Versoix (Kanton Genf), in der die Temperaturen auch im Sommer nur $13^{\circ} \mathrm{C}$ erreichen, zeigten die eingesetzten infizierten Sömmerlinge zwar einen Befall mit PKD, die Mortalität blieb jedoch unterhalb $10 \%$, einem Wert, der im Normalbereich liegt. In der wärmeren Langete (Kanton Bern), in der die kritische Temperaturschwelle bereits im Juni erstmals, und dann für längere Zeit ab Mitte Juli bis fast durchgehend Mitte September, überschritten wurde, starben um die $90 \%$ der eingesetzten infizierten Sömmerlinge (Schubiger et al., in Vorbereitung). Unsere Studien zur Verbreitung der PKD wiesen diese Krankheit in 49 der untersuchten 91 Flüsse nach (Wahli et al. 2007), was die weite Verbreitung der Krankheit in den Schweizer Fliessgewässern bestätigt (Wahli et al. 2002). In der früheren Studie wurde auch die Höhenverbreitung analysiert und es zeigte sich, dass ein Ausbruch der PKD in der Regel nur an Stellen beobachtet wurde, die unterhalb 800 Höhenmeter lagen und damit einem Bereich der mittleren Sommertemperatur von über $12^{\circ} \mathrm{C}\left( \pm 5^{\circ} \mathrm{C}\right)$ entsprechen (Hari et al. 2006). Wie stark sich die Krankheit auf den Bestand auswirkt, lässt sich an ihrer Bedeutung für die Sömmerlingsabundanz ablesen: Diese ist signifikant negativ mit dem Vorkommen der PKD im jeweiligen Gewässer korreliert (Schager et al. 2007). Dies war einer der überzeugenden Hinweise für den massgeblichen Einfluss dieser Krankheit am Forellenrückgang.

\subsection{Habitatverschiebung}

Eine andere Folge des Temperaturanstiegs hängt mit der Bevorzugung sommerkühler Gewässer zusammen: Bei höheren Temperaturen gerät die Bachforelle in Konkurrenz mit wärmetoleranteren Arten, wie Äsche und Barbe. Für die Rocky Mountains wurde berechnet, dass ein Anstieg der mittleren Juli-Temperaturen um $1{ }^{\circ} \mathrm{C}$ eine Verkleinerung der Bachforellenregion um 17\% zur Folge hätte (Rahel et al. 1996). Nach unseren Daten verschlechtert sich die Situation für Bachforellen im Alpenraum in Gewässerabschnitten unterhalb $600 \mathrm{~m}$ Höhe, während die Bachforellen in Gewässern oberhalb von 600 Höhenmetern bessere Temperaturbedingungen vorfinden. Unseren Berechnungen zufolge verschiebt der Anstieg der Wassertemperaturen den Lebensraum der Forellen bis zu 130 Höhenmeter nach flussaufwärts (Hari et al. 2006). Diese Verlagerung der Forellenregion stromaufwärts mit einer Ausweitung der sich daran anschliessenden Fischregionen ist aber angesichts der ausserordentlich zahlreichen Verbauungen in der Praxis nicht oder nur sehr eingeschränkt möglich (Fischnetz 2004; Matulla et al. 2007). Die 88.000 künstlichen, über $50 \mathrm{~cm}$ hohen Durchgangsbarrieren können nur von den wenigen, sprungkräftigen Forellen überwunden werden (Notter et al. 2007).

\subsection{Vermehrte Hochwasser}

Der Zeitpunkt der Hochwasserereignisse ist für die aus dem Kies aufsteigenden Bachforellenbrütlinge besonders kritisch, da sie erst eingeschränkt schwimmfähig sind und so über ein noch wenig ausgeprägtes Fluchtvermögen verfügen (Berry et al. 2003). Frühere Frühlingshochwasser können für diese Brütlinge fatale Auswirkungen haben, wenn sie mit dem Zeitpunkt ihrer Emergenz zusammenfallen.

Das Auswaschen der Eier wird hingegen nur bei starken Hochwassern angenommen. Von diesen wird nur in einzelnen Einzugsgebieten wie der Emme eine Häufigkeit und Stärke erwartet, die durch Geschiebetrieb und Sedimentverlagerung an der Flusssohle einen negativen Effekt auf die Eientwicklung bewirken (Santschi 2003).

\subsection{Erosion und Feinsedimenteintrag -} ihre Wirkung auf Gesundheit und Reproduktionserfolg

Treten in einem Fliessgewässer erhöhte Schwebstoffkonzentrationen auf, weichen die Fische vorübergehend in Unterstände oder unbelastete Seitengewässer aus. Wenn dies nicht möglich ist und die Tiere den erhöhten Konzentrationen ausgesetzt bleiben, reagieren die Fische unterschiedlich.

In Laborstudien wurde gezeigt, dass Schwebstoffe bei Werten von $90 \mathrm{mg} \mathrm{l}^{-1}$ und mehr das Risiko für Mortalität und Schäden an Kiemen und Haut erhöhen und der Fisch mit Anzeichen subletalen Stress', wie Veränderungen in der Blutchemie, antwortet. Dazu kommen Verhaltensänderungen wie reduzierte Nahrungsaufnahme, Vermeideverhalten und anderes (zusammengestellt in Scheurer et al. 2009). Feldstudien fehlen jedoch weitgehend. Im Freiland sind niedrigere Konzentrationen über längere Zeiträume oder diskontinuierliche Expositionsmuster vorherrschend. Ausserdem ist zu erwarten, dass die unterschiedliche geochemische Beschaffenheit und Grösse der Sedimentpartikel erheblichen Einfluss auf das Ausmass der Schadwirkung hat: Eckige Partikel rufen bereits bei niedrigeren Konzentrationen stärkere Schädigungen hervor als runde Partikel bei höheren Konzentrationen (Bilotta und Brazier 2008).

Die berechneten $80 \%$ Percentile der Schwebstoffe (14-Tage-Proben) in den Fliessgewässern der Schweiz liegen meist unter $100 \mathrm{mg} \mathrm{l}^{-1}$, höhere Werte werden vor allem in alpinen Gewässern während der Sommermonate gemessen oder stellen lokale Ereignisse (z. B. infolge Stauraumspülungen) dar (Fischnetz 2004). Wir müssen dennoch mit gewissen gesundheitlichen Beeinträchtigungen rechnen, besonders unter Berücksichtigung der im Ökosystem vorherrschenden längeren oder diskontinuierlichen Belastungen und der Kombination mit anderen Stressfaktoren. Mit der Zunahme von Starkregenereignissen im Frühjahr und 
der früheren Emergenz der Brütlinge aus dem Kies sind zudem die besonders empfindlichen Lebensstadien den Hochwassern und den damit auftretenden Schwebstofffrachten ausgesetzt.

\section{Diskussion: Zusammenhang zwischen Forellenrückgang und Klimaveränderung}

Im vorliegenden Artikel wurde dargestellt, inwiefern Temperaturanstieg, Abfluss und Hochwasser und Feinsedimenteintrag durch die Klimaveränderung beeinflusst werden, und welche Wirkung diese Faktoren auf die Bachforelle haben.

Der Temperaturanstieg in allen Gewässern der Schweiz um etwa $1{ }^{\circ} \mathrm{C}$ in den letzten 25 Jahren, mit einem deutlichen Sprung in den Jahren 1986/87, führt zu einer Verschlechterung der Situation für Bachforellen, die sommerkühle Gewässer bevorzugen. Dies trifft vor allem für die unteren Höhenlagen ihres Verbreitungsgebietes zu, während sich an den oberen Rändern der Verbreitungszone eine Verbesserung der temperaturabhängigen Rahmenbedingungen abzeichnet. Dementsprechend wurde die Fangstatistik hinsichtlich der Höhe über dem Meer auf einen möglichen Zusammenhang zwischen Fang und Klimaveränderung hin analysiert: Es wird deutlich, dass die Fangabnahme umso geringer ausfällt, je höher die Fangstrecken gelegen sind. Während die Fänge zwischen 1000 und $1400 \mathrm{~m}$ kaum abgenommen, in einigen Fliessgewässern sogar zugenommen haben, sinken die Fänge in den tiefer gelegenen Flussabschnitten im Untersuchungszeitraum rapide (Hari et al. 2006). Dazu kommen weitere anthropogene Einflüsse: Die Beseitigung der Ufervegetation reduziert die Beschattung und trägt damit zur Temperaturerhöhung bei. Veränderungen im Abflussregime, zu denen neben den Stauhaltungen auch Drainagen zu zählen sind, und die Einleitungen von erwärmtem Abwässern aus Industrie, Haushalten und Kläranlagen, führen ebenfalls zu einem Anstieg der Wassertemperaturen in den Fliessgewässern (Umwelt 2007). In der Rhone konnte man die Veränderung des Temperaturverlaufs durch die Stauhaltungen mit einer Erhöhung im Winter um bis zu $2{ }^{\circ} \mathrm{C}$ und einer Abkühlung im Sommer um $1{ }^{\circ} \mathrm{C}$ feststellen (Meier und Wüest 2004).

Die Temperaturerhöhung geschieht nicht während des ganzen Jahres gleichmässig, sondern betrifft vor allem die erste Jahreshälfte. Dadurch wird der Schlüpfzeitpunkt der Bachforellen um etwa 14 Tage vorverschoben (Hari et al. 2006), die Konsequenzen für die Entwicklung und Überlebensfähigkeit sind indes noch nicht absehbar. Die Temperaturerhöhung hat weitere indirekte Effekte, z.B. auf die Verbreitung temperaturabhängiger Fischkrankheiten wie der Nierenkrankheit PKD. Auch die Furunkulose, eine bakterielle Forellenkrankheit, bricht erst bei einer Temperatur oberhalb von $14^{\circ} \mathrm{C}$ aus und hat hohe Mortalitäten zur Folge (Fischnetz 2004).

Die Klimaveränderung wirkt sich auf Niederschlagsmenge, -stärke und -verteilung (weniger Schnee, der früher taut, mehr Starkregenereignisse, mehr Hochwasser im Winter und frühen Frühjahr) aus. Das wiederum führt zu vermehrtem Hochwasser und erhöht die Erosion, was eine Zunahme des Feinsedimenteintrags in die Flüsse mit sich bringen wird. Daneben führt auch die hydroelektrische Nutzung, insbesondere bei Stauhaltungen und Schwallbetrieb, zu extremen Abflussschwankungen und erhöht ebenfalls zeitweise die Fracht an Schwebstoffen und Sedimenten. In derartigen Strecken konnte eine signifikante Beeinträchtigung der Fischpopulationen festgestellt werden (Weber et al. 2007). Auch Veränderungen in der land- und forstwirtschaftlichen Flächennutzung (z.B. vermehrtes Brachliegen von Alpenwiesen, Intensivierung der Landwirtschaft, vermehrter Anbau nicht-bodendeckender Feldfrüchte wie Mais) tragen zu einer Verstärkung der Erosion bei. Die Ablagerung von Feinsedimentpartikeln führt zu einer erhöhten Kolmation der Bachsohle. Dies wird begünstigt durch Meliorationen, Uferbegradigungen und Verbauungen von Flüssen, die zu einer Verstetigung des Abflusses beitragen und eine Kolmation fördern.

Ausserdem führt das veränderte Niederschlagsmuster zu vermehrten Hochwassern während der Zeit der Emergenz der Brütlinge. Diese sind zugleich kritische Lebensstadien, die aufgrund ihrer noch wenig ausgebildeten Schwimmfähigkeit einem erhöhten Abfluss nur begrenzt durch Ausweichen in andere Habitate begegnen können. Durch die Fragmentierungen und Monotonisierung der Gewässer sind Rückzugsmöglichkeiten für empfindliche Lebensstadien zusätzlich reduziert.

\section{Schlussfolgerungen}

In den zunehmend wärmeren Mittellandflüssen ist zukünftig mit einer Abnahme des Bachforellenfangertrags und einer Zunahme von Fischkrankheiten zu rechnen. Die Bachforelle wird hier zudem durch wärmeliebendere - oder weniger wärmeempfindlichere Arten - konkurrenziert. Je nach Fliessgewässer sind unterschiedliche Folgen des Temperaturanstiegs zu erwarten: In sommerkühlen Gewässern im alpinen Raum oder solchen, die durch Gletscherwasser gespeist werden, sind dank des Temperaturanstiegs über eine längere Zeit des Jahres für die Bachforelle optimale Temperaturen zu erwarten. In Gewässern des Mittellandes, die sich bereits an der Verbreitungsgrenze der Bachforelle befinden, verkürzt sich die Zeitdauer optimaler Wassertemperaturen für die Bachforelle weiter.

Empfehlungen für Massnahmen zur Reduktion nachteiliger Wirkungen der Klimaveränderung sind aus der Warte 
eines Anwalts der Fische folgendermassen zu formulieren: Bei Revitalisierungen ist die Aufforstung mit standortgerechten Ufergehölzen anzustreben, um eine Beschattung zu gewährleisten und einer übermässigen Aufwärmung der Bäche in Hitzeperioden entgegenzuwirken. Die Lebensräume für die Fische sind zu verbessern und zu vernetzen. Die Wiederanbindung von Seitengewässern und der Oberläufe der Flüsse erlaubt die Nutzung dieser vielfältigen Lebensräume und sichert dadurch eine Habitatvielfalt, die besonders für anspruchsvolle Fischarten wie die Bachforelle wichtig ist.

Danksagung Für Beiträge und Diskussionen danke ich Nicolas Biber, Fynn Holm, Christian Michel, Sara Schubert und Karen L. Thorpe, sowie allen Beteiligten im Projekt Fischnetz für die zugrundeliegenden Daten und Diskussionen.

\section{Literatur}

Asselman NEM, Middelkoop H, van Dijk PM (2003) The impact of changes in climate and land use on soil erosion, transport and deposition of suspended sediment in the river Rhine. Hydrol Proc 17:3225-3244

Bader S, Kunz P(1998) Klimarisiken-Herausforderung für die Schweiz. Wissenschaftlicher Schlussbericht NFP 31, Zürich, $307 \mathrm{~S}$

Berry W, Rubinstein N, Melzia B, Hill B (2003) The biological effects of suspended and bedded sediment (SABS) in aquatic systems: a review. USEPA, Washington DC, $58 \mathrm{pp}$

Bilotta GS, Brazier RE (2008) Understanding the influence of suspended solids on water quality and aquatic biota. Water Res 42(12):2849-2861

Birsan MV, Molnar P, Burlando P, Pfaundler M (2005) Streamflow trends in Switzerland. J Hydrol 314:312-329

Brunetti M, Maugeri M, Nanni T, Auer I, Bohm R, Schoner W (2006) Precipitation variability and changes in the greater alpine region over the 1800-2003 period. J Geophys Res [Atmos] 111, D11107. DOI: 10.1029/2005JD006674

Burkhardt-Holm P, Giger W, Güttinger H, Ochsenbein U, Peter A, Scheurer K, Segner H, Staub E, Suter MJF (2005) Where have all the fish gone? Env Sci Technol 39: 441A-447A

Burkhardt-Holm P, Peter A, Segner H (2002) Decline of fish catch in Switzerland - Project Fishnet: A balance between analysis and synthesis. Aquat Sci 64:36-54

Crisp (2000) Trout and Salmon: ecology, conservation and rehabilitation. Blackwell Science, Oxford, $212 \mathrm{~S}$

Elliott JM (1981) Some aspects of thermal stress on freshwater teleosts. In: Pickering AD (ed) Stress and fish. Academic Press, London, pp 209-245

Feist SW, Bucke D (1993) Proliferative kidney disease in wild salmonids. Fish Res 17:51-58

Fischnetz (2004) Dem Fischrückgang auf der Spur. Schlussbericht des Projekts Netzwerk Fischrückgang Schweiz ,Fischnetz'. EAWAG, Bundesamt für Umwelt, Wald und Landschaft, Bern, $178 \mathrm{~S}$

Greig SM, Sear DA, Carling PA (2007) A review of factors influencing the availability of dissolved oxygen to incubating salmonid embryos. Hydrol Proc 21:323-334

Hari RE, Livingstone DM, Siber R, Burkhardt-Holm P, Güttinger H (2006) Consequences of climatic change for water temperature and brown trout populations in alpine rivers and streams. Glob Change Biol 12:10-26

Horton P, Schaefli B, Mezghani A, Hingray B, Musy A (2006) Assessment of climate-change impacts on alpine discharge regimes with climate model uncertainty. Hydrol Processes 20:2091-2109
Jasper K, Calanca P, Gyalistras D, Fuhrer J (2004) Differential impacts of climate change on the hydrology of two alpine river basins. Climate Res 26:113-129

Matulla C, Schmutz S, Melcher A, Gerersdorfer T, Haas P (2007) Assessing the impact of a downscaled climate change simulation on the fish fauna in an Inner-Alpine River. Int J Biometerol $52: 127-137$

Meier W, Wüest A (2004) Wie verändert die hydroelektrische Nutzung die Wassertemperatur der Rhone? Wasser Energie Luft 96:305-309

Mohseni O, Stefan HG (1999) Stream temperature/air temperature relationship: a physical interpretation. J Hydrol 218:128-141

Notter B, Aschwanden H, Klauser H, Staub E, von Blücher U (2007) Ökomorphologischer Zustand der Schweizer Fliessgewässer: Zwischenauswertung aufgrund der Erhebungen aus 18 Kantonen, Bafu, Mediendienst, S 1-9

Perry AL, Low PL, Ellis JR, Reynolds JD (2005) Climate Change and Distribution Shifts in Marine Fishes. Science 308:1912

Peter A (1998) Interruption of the river continuum by barriers and the consequences for migratory fish. In: Jungwirth M, Schmutz S, Weiss $\mathrm{S}$ (eds) Fish migration and fish bypasses. Fishing News Books, Oxford, pp 99-112

Rahel FJ, Keleher CJ, Anderson JL (1996) Potential habitat loss and population fragmentation for cold water fish in the North Platte River drainage of the Rocky Mountains: response to climate warming. Limnology and Oceanography 41:1116-1123

Reichenbach-Klinke H-HE (1976) Die Gewässeraufheizung und ihre Auswirkung auf den Lebensraum Wasser. In: Fisch und Umwelt, Gustav Fischer Verlag, Stuttgart, S 153-161

Rombough PJ (1997) The effects of temperature on embryonic and larval development. In: Wood CM, McDonald DG (eds) Global warming: implications for freshwater and marine fish. Cambridge University Press, Cambridge, pp 177-223

Roussel JM, Bardonnet A (2002) The habitat of juvenile brown trout (Salmo trutta L.) in small streams: Preferences, movements, diel and seasonal variations. Bulletin français de la pêche et de la pisciculture 365-66:435-454

Santschi D (2003) Zeitliche Veränderung der winterlichen Abflusscharakteristik schweizerischer Fliessgewässer. Diplomarbeit, Universität Bern, Bern, $109 \mathrm{~S}$

Schädler B (2000) Klimaveränderung und Naturkatastrophen in der Schweiz. Klimaveränderung und Konsequenzen für die Wasserwirtschaft. KLIWA Symposium, Karlsruhe. Arbeitskreis KLIWA, S 204-211

Scheurer K, Alewell C, Bänninger D, Burkhardt-Holm P (2009) Climate and land-use changes affecting river sediment and brown trout in alpine countries-a review. Env Sci Pollut Res, DOI: 10.1007/s11356-008-0075-3

Schmeing-Engberding F (1953) Die Vorzugstemperaturen einiger Knochenfische und ihre physiologische Bedeutung. Zeitschrift für Fischerei und deren Hilfswissenschaften 2:125-155

Schager E, Peter A, Burkhardt-Holm P (2007) Status of young-of-theyear brown trout (Salmo trutta fario) in Swiss streams. Factors influencing YOY trout recruitment. Aquat Sci 69:41-50

Schubiger C, Segner H, Wahli T (in Vorbereitung) Influence of temperature, fish origin and stocking season on the manifestation of the proliferative kidney disease (PKD) in brown trout (Salmo trutta fario)

Summer W, Zhang W, Striztinger W (1994) Consequences of human impacts on the sediment transport process. Z f Kulturtechnik und Landentwicklung 35:382-389

Umwelt Schweiz (2007) BAFU/BFS (Hrsg), Bern und Neuchâtel, $148 \mathrm{~S}$

Wahli T, Knuesel R, Bernet D, Segner H, Pugovkin D, BurkhardtHolm P, Escher M, Schmidt-Posthaus H (2002) Proliferative kidney disease in Switzerland: Current state of knowledge. J Fish Dis 25:491-500 
Wahli T, Bernet D, Steiner PA, Schmidt-Posthaus H (2007) Geographic distribution of Tetracapsuloides bryosalmonae infected fish in Swiss rivers: an update. Aquat Sci 69:3-10

Weber C, Peter A, Zanini F (2007) Spatio-temporal analysis of fish and their habitat: a case study on a highly degraded Swiss river system prior to extensive rehabilitation. Aquat Sci 69:162-172
Zaugg B, Stucki P, Pedroli J-C \& Kirchhofer A (2003) Fauna Helvetica. Pisces Atlas. Centre suisse de cartographie de la faune (CSCF/ SZKF), Bern, $233 \mathrm{~S}$

Zobrist J, Sigg L, Schönenberger U (2004) NADUF - Thematische Auswertung der Messresultate 1974-1998. Schriftenreihe Nr. 18, EAWAG, Dübendorf, $125 \mathrm{~S}$ 